\title{
Some properties of semi-tensor bundle
}

\author{
Murat Polat* and Nejmi Cengiz \\ Department of Mathematics, Faculty of Science, Atatürk University, Erzurum, Turkey \\ Received: 19 January 2018, Accepted: 10 June 2018 \\ Published online: 10 August 2018.
}

\begin{abstract}
Using the fiber bundle $\mathrm{M}$ over a manifold B, we define a semi-tensor (pull-back) bundle tB of type (p,q). The present paper is devoted to some results concerning with the vertical and complete lifts of some tensor fields from manifold $\mathrm{B}$ to its semi-tensor bundle $\mathrm{tB}$ of type $(\mathrm{p}, \mathrm{q})$.
\end{abstract}

Keywords: Vector field, complete lift, pull-back bundle, semi-tensor bundle.

\section{Introduction}

Let $M_{n}$ be an $n$-dimensional differentiable manifold of class $C^{\infty}$ and $\pi_{1}: M_{n} \rightarrow B_{m}$ the differentiable bundle determined by a submersion $\pi_{1}$. Suppose that $\left(x^{i}\right)=\left(x^{a}, x^{\alpha}\right), a, b, \ldots=1, \ldots, n-m ; \alpha, \beta, \ldots=n-m+1, \ldots, n ; i, j, \ldots=1,2, \ldots, n$ is a system of local coordinates adapted to the bundle $\pi_{1}: M_{n} \rightarrow B_{m}$, where $x^{\alpha}$ are coordinates in $B_{m}$, and $x^{a}$ are fiber coordinates of the bundle $\pi_{1}: M_{n} \rightarrow B_{m}$. If $\left(x^{i^{\prime}}\right)=\left(x^{a^{\prime}}, x^{\alpha^{\prime}}\right)$ is another system of local adapted coordinates in the bundle, then we have [14]

$$
\left\{\begin{array}{l}
x^{a^{\prime}}=x^{a^{\prime}}\left(x^{b}, x^{\beta}\right), \\
x^{\alpha^{\prime}}=x^{\alpha^{\prime}}\left(x^{\beta}\right) .
\end{array}\right.
$$

The Jacobian of (1) has components

$$
\left(A_{j}^{i^{\prime}}\right)=\left(\frac{\partial x^{i^{\prime}}}{\partial x^{j}}\right)=\left(\begin{array}{cc}
A_{b}^{a^{\prime}} & A_{\beta}^{a^{\prime}} \\
0 & A_{\beta}^{\alpha^{\prime}}
\end{array}\right),
$$

where

$$
A_{\beta}^{\alpha^{\prime}}=\frac{\partial x^{\alpha^{\prime}}}{\partial x^{\beta}} .
$$

Let $\left(T_{q}^{p}\right)_{x}\left(B_{m}\right)\left(x=\pi_{1}(\widetilde{x}), \widetilde{x}=\left(x^{a}, x^{\alpha}\right) \in M_{n}\right)$ be the tensor space at a point $x \in B_{m}$ with local coordinates $\left(x^{1}, \ldots, x^{m}\right)$, we have the holonomous frame field

$$
\partial_{x^{i_{1}}} \otimes \partial_{x^{i_{2}}} \otimes \ldots \otimes \partial_{x^{i_{p}}} \otimes d x^{j_{1}} \otimes d x^{j_{2}} \otimes \ldots \otimes d x^{j_{q}}
$$

for $i \in\{1, \ldots, m\}^{p}, j \in\{1, \ldots, m\}^{q}$, over $U \subset B_{m}$ of this tensor bundle, and for any $(p, q)$-tensor field $t$ we have [[4], p.163]:

$$
t \mid U=t_{j_{1} \ldots j_{q}}^{i_{1} \ldots i_{p}} \partial_{x^{i_{1}}} \otimes \partial_{x^{i_{2}}} \otimes \ldots \otimes \partial_{x^{i_{p}}} \otimes d x^{j_{1}} \otimes d x^{j_{2}} \otimes \ldots \otimes d x^{j_{q}}
$$

then by definition the set of all points $\left(x^{I}\right)=\left(x^{a}, x^{\alpha}, x^{\bar{\alpha}}\right), x^{\bar{\alpha}}=t_{j_{1} \ldots j_{q}}^{i_{1} \ldots i_{p}}, \bar{\alpha}=\alpha+m^{p+q}, I, J, \ldots=1, \ldots, n+m^{p+q}$ is a semi-tensor bundle $t_{q}^{p}\left(B_{m}\right)$ over the manifold $M_{n}$ [14]. The semi-tensor bundle $t_{q}^{p}\left(B_{m}\right)$ has the natural bundle structure over $B_{m}$, its 
bundle projection $\pi: t_{q}^{p}\left(B_{m}\right) \rightarrow B_{m}$ being defined by $\pi:\left(x^{a}, x^{\alpha}, x^{\bar{\alpha}}\right) \rightarrow\left(x^{\alpha}\right)$. If we introduce a mapping $\pi_{2}: t_{q}^{p}\left(B_{m}\right) \rightarrow M_{n}$ by $\pi_{2}:\left(x^{a}, x^{\alpha}, x^{\bar{\alpha}}\right) \rightarrow\left(x^{a}, x^{\alpha}\right)$, then $t_{q}^{p}\left(B_{m}\right)$ has a bundle structure over $M_{n}$. It is easily verified that $\pi=\pi_{1} \circ \pi_{2}$ [14].

On the other hand, let $\varepsilon=\pi: E \rightarrow B$ denote a fiber bundle with fiber $F$. Given a manifold $B^{\prime}$ and a map $f: B^{\prime} \rightarrow B$, one can construct in a natural way a bundle over $B^{\prime}$ with the same fiber: Consider the subset

$$
f^{*} E=\left\{\left(b^{\prime}, e\right) \in B^{\prime} \times E \mid f\left(b^{\prime}\right)=\pi(e)\right\}
$$

together with the subspace topology from $B^{\prime} \times E$, and denote by $\pi_{1}: f^{*} E \rightarrow B^{\prime}, \pi_{2}: f^{*} E \rightarrow E$ the projections. $f^{*} \varepsilon=\pi_{1}$ : $f^{*} E \rightarrow B^{\prime}$ is a fiber bundle with fiber $F$, called the pull-back bundle of $\varepsilon$ via $f$ [[3], [5], [8], [10], [14]].

From the above definition it follows that the semi-tensor bundle $\left(t_{q}^{p}\left(B_{m}\right), \pi_{2}\right)$ is a pull-back bundle of the tensor bundle over $B_{m}$ by $\pi_{1}$ (see, for example [12], [14]).

In other words, the semi-tensor bundle (induced or pull-back bundle) of the tensor bundle $\left(T_{q}^{p}\left(B_{m}\right), \tilde{\pi}, B_{m}\right)$ is the bundle $\left(t_{q}^{p}\left(B_{m}\right), \pi_{2}, M_{n}\right) \quad$ over $\quad M_{n} \quad$ with a $\quad$ a total space $t_{q}^{p}\left(B_{m}\right)=\left\{\left(\left(x^{a}, x^{\alpha}\right), x^{\bar{\alpha}}\right) \in M_{n} \times\left(T_{q}^{p}\right)_{x}\left(B_{m}\right): \pi_{1}\left(x^{a}, x^{\alpha}\right)=\tilde{\pi}\left(x^{\alpha}, x^{\bar{\alpha}}\right)=\left(x^{\alpha}\right)\right\} \subset M_{n} \times\left(T_{q}^{p}\right)_{x}\left(B_{m}\right)$.To a transformation (1) of local coordinates of $M_{n}$, there corresponds on $t_{q}^{p}\left(B_{m}\right)$ the coordinate transformation

$$
\left\{\begin{array}{l}
x^{a^{\prime}}=x^{a^{\prime}}\left(x^{b}, x^{\beta}\right) \\
x^{\alpha^{\prime}}=x^{\alpha^{\prime}}\left(x^{\beta}\right) \\
x^{\bar{\alpha}^{\prime}}=t_{\alpha_{1}^{\prime} \ldots \alpha_{q}^{\prime}}^{\beta_{1}^{\prime} \ldots \beta_{p}^{\prime}}=A_{\alpha_{1} \ldots \alpha_{p}}^{\beta_{1}^{\prime} \ldots \beta_{p}^{\prime}} A_{\alpha_{1}^{\prime} \ldots \alpha_{q}^{\prime}}^{\beta_{1}^{\prime} \ldots \beta_{q}} t_{\beta_{1} \ldots \beta_{q}}^{\alpha_{1} \ldots \alpha_{p}}=A_{(\alpha)}^{\left(\beta^{\prime}\right)} A_{\left(\alpha^{\prime}\right)}^{(\beta)} x^{\bar{\beta}} .
\end{array}\right.
$$

The Jacobian of (2) is given by [14]:

$$
\bar{A}=\left(A_{J}^{I^{\prime}}\right)=\left(\begin{array}{ccc}
A_{b}^{a^{\prime}} & 0 & 0 \\
0 & A_{\beta}^{\alpha^{\prime}} & 0 \\
0 & t_{(\sigma)}^{(\alpha)} \partial_{\beta} A_{(\alpha)}^{\left(\beta^{\prime}\right)} A_{\left(\alpha^{\prime}\right)}^{(\sigma)} A_{(\alpha)}^{\left(\beta^{\prime}\right)} A_{\left(\alpha^{\prime}\right)}^{(\beta)}
\end{array}\right)
$$

where $I=(a, \alpha, \bar{\alpha}), J=(b, \beta, \bar{\beta}), I, J \ldots=1, \ldots, n+m^{p+q}, t_{(\sigma)}^{(\alpha)}=t_{\sigma_{1} \ldots \sigma_{q}}^{\alpha_{1} \ldots \alpha_{p}}, A_{\beta}^{\alpha^{\prime}}=\frac{\partial x^{\alpha^{\prime}}}{\partial x^{\beta}}$.

It is easily verified that the condition $\operatorname{Det} \bar{A} \neq 0$ is equivalent to the condition:

$$
\operatorname{Det}\left(A_{b}^{a^{\prime}}\right) \neq 0, \operatorname{Det}\left(A_{\beta}^{\alpha^{\prime}}\right) \neq 0, \operatorname{Det}\left(A_{(\alpha)}^{\left(\beta^{\prime}\right)} A_{\left(\alpha^{\prime}\right)}^{(\beta)}\right) \neq 0 .
$$

Also, $\operatorname{dim} t_{q}^{p}\left(B_{m}\right)=n+m^{p+q}$. In the special case $n=m, t_{q}^{p}\left(B_{m}\right)$ is a tensor bundle $T_{q}^{p}\left(B_{m}\right)$ [[6], p.118]. In the special case, the semi-tensor bundles $t_{0}^{1}\left(B_{m}\right)(p=1, q=0)$ and $t_{1}^{0}\left(B_{m}\right)(p=0, q=1)$ are semi-tangent and semi-cotangent bundles, respectively. We note that semi-tangent and semi-cotangent bundle were examined in [[1], [7], [9]] and [[11], [13], [15], [16]], respectively. Also, Fattaev studied the special class of semi-tensor bundle [2]. We denote by $\mathfrak{J}_{q}^{p}\left(t_{q}^{p}\left(B_{m}\right)\right)$ and $\mathfrak{I}_{q}^{p}\left(B_{m}\right)$ the modules over $F\left(t_{q}^{p}\left(B_{m}\right)\right)$ and $F\left(B_{m}\right)$ of all tensor fields of type $(p, q)$ on $t_{q}^{p}\left(B_{m}\right)$ and $B_{m}$ respectively, where $F\left(t_{q}^{p}\left(B_{m}\right)\right)$ and $F\left(B_{m}\right)$ denote the rings of real-valued $C^{\infty}$-functions on $t_{q}^{p}\left(B_{m}\right)$ and $B_{m}$, respectively.

\section{Some lifts of tensor fields and $\gamma-$ operator}

Let $\widetilde{X} \in \mathfrak{I}_{0}^{1}\left(M_{n}\right)$ be a projectable vector field [9] with projection $X=X^{\alpha}\left(x^{\alpha}\right) \partial_{\alpha}$ i.e. $\widetilde{X}=\widetilde{X}^{a}\left(x^{a}, x^{\alpha}\right) \partial_{a}+X^{\alpha}\left(x^{\alpha}\right) \partial_{\alpha}$. On putting

$$
{ }^{c c} \tilde{X}=\left(\begin{array}{l}
{ }^{c c} \widetilde{X}^{b} \\
{ }^{c c} \widetilde{X}^{\beta} \\
{ }^{c} \widetilde{X}^{\beta}
\end{array}\right)=\left(\begin{array}{l}
\widetilde{X}^{b} \\
\widetilde{X}^{\beta} \\
\sum_{\lambda=1}^{p} t_{\beta_{1} \ldots \beta_{q}}^{\alpha_{1} \ldots \varepsilon \ldots \alpha_{p}} \partial_{\varepsilon} X^{\beta_{\lambda}}-\sum_{\mu=1}^{q} t_{\beta_{1} \ldots \varepsilon \ldots \beta_{q}}^{\alpha_{1} \ldots \alpha_{p}} \partial_{\beta_{\mu}} X^{\varepsilon}
\end{array}\right),
$$


we easily see that ${ }^{c c} \widetilde{X}^{\prime}=\bar{A}\left({ }^{c c} \widetilde{X}\right)$. The vector field ${ }^{c c} \widetilde{X}$ is called the complete lift of $\widetilde{X}$ to the semi-tensor bundle $t_{q}^{p}\left(B_{m}\right)$ [14].

Now, consider $A \in \mathfrak{I}_{q}^{p}\left(B_{m}\right)$ and $\varphi \in \mathfrak{I}_{1}^{1}\left(B_{m}\right)$, then ${ }^{v v} A \in \mathfrak{I}_{0}^{1}\left(t_{q}^{p}\left(B_{m}\right)\right)$ (vertical lift), $\gamma \varphi \in \mathfrak{I}_{0}^{1}\left(t_{q}^{p}\left(B_{m}\right)\right)$ and $\tilde{\gamma} \varphi \in \mathfrak{I}_{0}^{1}\left(t_{q}^{p}\left(B_{m}\right)\right)$ have respectively, components on the semi-tensor bundle $t_{q}^{p}\left(B_{m}\right)[14]$

$$
{ }^{v v} A=\left(\begin{array}{l}
0 \\
0 \\
A_{\beta_{1} \ldots \beta_{q}}^{\alpha_{1} \ldots \alpha_{p}}
\end{array}\right), \gamma \varphi=\left(\begin{array}{l}
0 \\
0 \\
\sum_{\lambda=1}^{p} t_{\beta_{1} \ldots \beta_{q}}^{\alpha_{1} \ldots \varepsilon \ldots \alpha_{p}} \varphi_{\varepsilon}^{\alpha_{\lambda}}
\end{array}\right), \tilde{\gamma} \varphi=\left(\begin{array}{l}
0 \\
0 \\
\sum_{\mu=1}^{q} t_{\beta_{1} \ldots \varepsilon \ldots \beta_{q}}^{\alpha_{1} \ldots \alpha_{p}} \varphi_{\beta_{\mu}}^{\varepsilon}
\end{array}\right)
$$

with respect to the coordinates $\left(x^{a}, x^{\alpha}, x^{\bar{\alpha}}\right)$ on $t_{q}^{p}\left(B_{m}\right)$, where $A_{\beta_{1} \ldots \beta_{q}}^{\alpha_{1} \ldots \alpha_{p}}, \varphi_{\varepsilon}^{\alpha_{\lambda}}$ and $\varphi_{\beta_{\mu}}^{\varepsilon}$ are local components of $A$ and $\varphi$.

On the other hand, ${ }^{v v} f$ the vertical lift of function $f \in \mathfrak{I}_{0}^{0}\left(B_{m}\right)$ on $t_{q}^{p}\left(B_{m}\right)$ is defined by [14]:

$$
{ }^{v v} f={ }^{v} f \circ \pi_{2}=f \circ \pi_{1} \circ \pi_{2}=f \circ \pi .
$$

Theorem 1. For any vector fields $\widetilde{X}, \widetilde{Y}$ on $M_{n}$ and $f \in \mathfrak{I}_{0}^{0}\left(B_{m}\right)$, we have

(i) ${ }^{c c}(\widetilde{X}+\widetilde{Y})={ }^{c c} \widetilde{X}+{ }^{c c} \widetilde{Y}$,

(ii) ${ }^{c} \widetilde{X}^{v v} f={ }^{v v}(X f)$.

Proof. (i) This immediately follows from (4).

(ii) Let $\widetilde{X} \in \mathfrak{I}_{0}^{1}\left(M_{n}\right)$. Then we get by (4) and (6):

$$
{ }^{c c} \widetilde{X}^{v v} f={ }^{c c} \widetilde{X}^{I} \partial_{I}\left({ }^{v v} f\right)={ }^{c c} \widetilde{X}^{a} \underbrace{\partial_{a}\left({ }^{v v} f\right)}_{0}+{ }^{c c} \widetilde{X}^{\alpha} \partial_{\alpha}\left({ }^{v v} f\right)+{ }^{c c} \widetilde{X}^{\bar{\alpha}} \underbrace{\partial_{\bar{\alpha}}\left({ }^{v v} f\right)}_{0}=X^{\alpha} \partial_{\alpha}\left({ }^{v v} f\right)={ }^{v v}(X f),
$$

which gives (ii) of Theorem 1.

Theorem 2. If $\varphi \in \mathfrak{I}_{1}^{1}\left(B_{m}\right), f \in \mathfrak{I}_{0}^{0}\left(B_{m}\right)$ and $A \in \mathfrak{I}_{q}^{p}\left(B_{m}\right)$, then

(i) $\left({ }^{v v} A\right)^{v v} f=0$,

(ii) $(\gamma \varphi)\left({ }^{v v} f\right)=0$,

(iii) $(\widetilde{\gamma} \varphi)\left({ }^{v v} f\right)=0$.

Proof. (i) If $A \in \mathfrak{I}_{q}^{p}\left(B_{m}\right)$, then, by (5) and (6), we find

$$
\left({ }^{v v} A\right)^{v v} f=\left({ }^{v v} A\right)^{I} \partial_{I}\left({ }^{v v} f\right)=\left({ }^{v v} A\right)^{a} \underbrace{\partial_{a}\left({ }^{v v} f\right)}_{0}+\underbrace{\left({ }^{v v} A\right)^{\alpha}}_{0} \partial_{\alpha}\left({ }^{v v} f\right)+\left({ }^{v v} A\right)^{\bar{\alpha}} \underbrace{\partial_{\bar{\alpha}}\left({ }^{v v} f\right)}_{0}=0 .
$$

Thus, we have $(i)$ of Theorem 2.

(ii) If $\varphi \in \mathfrak{I}_{1}^{1}\left(B_{m}\right)$, then we have by (5) and (6):

$$
(\gamma \varphi)\left({ }^{v v} f\right)=(\gamma \varphi)^{I} \partial_{I}\left({ }^{v v} f\right)=(\gamma \varphi)^{a} \underbrace{\partial_{a}\left({ }^{v v} f\right)}_{0}+\underbrace{(\gamma \varphi)^{\alpha}}_{0} \partial_{\alpha}\left({ }^{v v} f\right)+(\gamma \varphi)^{\bar{\alpha}} \underbrace{\partial_{\bar{\alpha}}\left({ }^{v v} f\right)}_{0}=0 .
$$

Thus, we have (ii) of Theorem 2.

(iii) If $\varphi \in \mathfrak{I}_{1}^{1}\left(B_{m}\right)$, then we have by (5) and (6):

$$
(\widetilde{\gamma} \varphi)\left({ }^{v v} f\right)=(\widetilde{\gamma} \varphi)^{I} \partial_{I}\left({ }^{v v} f\right)=(\widetilde{\gamma} \varphi)^{a} \underbrace{\partial_{a}\left({ }^{v v} f\right)}_{0}+\underbrace{(\widetilde{\gamma} \varphi)^{\alpha}}_{0} \partial_{\alpha}\left({ }^{v v} f\right)+(\widetilde{\gamma} \varphi)^{\bar{\alpha}} \underbrace{\partial_{\bar{\alpha}}\left({ }^{v v} f\right)}_{0}=0 .
$$


Thus, we have (iii) of Theorem 2.

Theorem 3. Let $A, B \in \mathfrak{I}_{q}^{p}\left(B_{m}\right)$. For the Lie product, we have

$$
\left[{ }^{v v} A,{ }^{v v} B\right]=0
$$

Proof. If $A, B \in \mathfrak{I}_{q}^{p}\left(B_{m}\right)$ and $\left(\begin{array}{c}{\left[{ }^{v v} A,{ }^{v v} B\right]^{b}} \\ {\left[{ }^{v v} A,{ }^{v v} B\right]} \\ {\left[{ }^{v v} A,{ }^{v} B\right]^{\beta}}\end{array}\right)$ are components of $\left[{ }^{v v} A,{ }^{v v} B\right]^{J}$ with respect to the coordinates $\left(x^{b}, x^{\beta}, x^{\bar{\beta}}\right)$ on $t_{q}^{p}\left(B_{m}\right)$, then we have

$$
\begin{aligned}
{\left[{ }^{v v} A,{ }^{v v} B\right]^{J} } & =\left({ }^{v v} A\right)^{I} \partial_{I}\left({ }^{v v} B\right)^{J}-\left({ }^{v v} B\right)^{I} \partial_{I}\left({ }^{v v} A\right)^{J} \\
& =\underbrace{\left({ }^{v v} A\right)^{a}}_{0} \partial_{a}\left({ }^{v v} B\right)^{J}+\underbrace{\left({ }^{v v} A\right)^{\alpha}}_{0} \partial_{\alpha}\left({ }^{v v} B\right)^{J}+\left({ }^{v v} A\right)^{\bar{\alpha}} \partial_{\bar{\alpha}}\left({ }^{v v} B\right)^{J}-\underbrace{\left({ }^{v v} B\right)^{a}}_{0} \partial_{a}\left({ }^{v v} A\right)^{J}-\underbrace{\left({ }^{v v} B\right)^{\alpha}}_{0} \partial_{\alpha}\left({ }^{v v} A\right)^{J}-\left({ }^{v v} B\right)^{\bar{\alpha}} \partial_{\bar{\alpha}}\left({ }^{v v} A\right)^{J} \\
& =A_{\beta_{1} \ldots \beta_{q}}^{\alpha_{1} \ldots \alpha_{p}} \partial_{\bar{\alpha}}\left({ }^{v v} B\right)^{J}-B_{\beta_{1} \ldots \beta_{q}}^{\alpha_{1} \ldots \alpha_{p}} \partial_{\bar{\alpha}}\left({ }^{v v} A\right)^{J} .
\end{aligned}
$$

Firstly, if $J=b$, we have

$$
\left[{ }^{v v} A,{ }^{v v} B\right]^{b}=A_{\beta_{1} \ldots \beta_{q}}^{\alpha_{1} \ldots \alpha_{p}} \partial_{\bar{\alpha}} \underbrace{\left({ }^{v v} B\right)^{b}}_{0}-B_{\beta_{1} \ldots \beta_{q}}^{\alpha_{1} \ldots \alpha_{p}} \partial_{\bar{\alpha}} \underbrace{\left({ }^{v v} A\right)^{b}}_{0}=0
$$

by virtue of (5). Secondly, if $J=\beta$, we have

$$
\left[{ }^{v v} A,{ }^{v v} B\right]^{\beta}=A_{\beta_{1} \ldots \beta_{q}}^{\alpha_{1} \ldots \alpha_{p}} \partial_{\bar{\alpha}} \underbrace{\left({ }^{v v} B\right)^{\beta}}_{0}-B_{\beta_{1} \ldots \beta_{q}}^{\alpha_{1} \ldots \alpha_{p}} \partial_{\bar{\alpha}} \underbrace{\left({ }^{v v} A\right)^{\beta}}_{0}=0
$$

by virtue of (5). Thirdly, if $J=\bar{\beta}$, then we have

$$
\left[{ }^{v v} A,{ }^{v v} B\right]^{\bar{\beta}}=A_{\beta_{1} \ldots \beta_{q}}^{\alpha_{1} \ldots \alpha_{p}} \partial_{\bar{\alpha}}\left({ }^{v v} B\right)^{\bar{\beta}}-B_{\beta_{1} \ldots \beta_{q}}^{\alpha_{1} \ldots \alpha_{p}} \partial_{\bar{\alpha}}\left({ }^{v v} A\right)^{\bar{\beta}}=A_{\beta_{1} \ldots \beta_{q}}^{\alpha_{1} \ldots \alpha_{p}} \underbrace{\partial_{\bar{\alpha}} B_{\theta_{1} \ldots \theta_{q}}^{\beta_{1} \ldots \beta_{p}}}_{0}-B_{\beta_{1} \ldots \beta_{q}}^{\alpha_{1} \ldots \alpha_{p}} \underbrace{\partial_{\bar{\alpha}} A_{\theta_{1} \ldots \theta_{q}}^{\beta_{1} \ldots \beta_{p}}}_{0}=0
$$

by virtue of (5). Thus, we have Theorem 3 .

Theorem 4. Let $\widetilde{X}$ and $\widetilde{Y}$ be projectable vector fields on $M_{n}$ with projections $X$ and $Y$ on $B_{m}$, respectively. For the Lie product, we have

$$
\left[{ }^{c c} \widetilde{X},{ }^{c c} \widetilde{Y}\right]={ }^{c c}[\widetilde{X}, \widetilde{Y}]\left(i \cdot e \cdot L_{c c}^{c c} \widetilde{Y}={ }^{c c}\left(L_{\widetilde{X}} \widetilde{Y}\right)\right)
$$

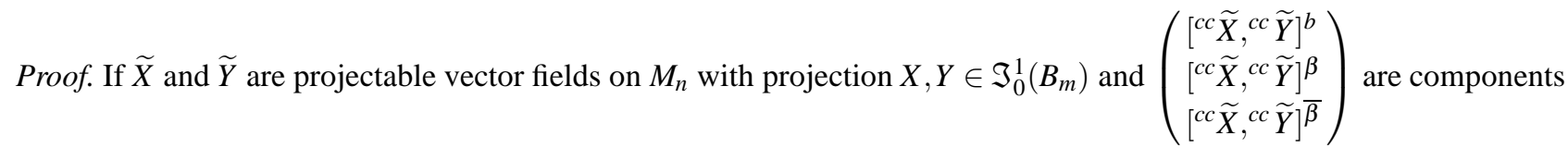
of $\left[{ }^{c c} \widetilde{X},{ }^{c c} \widetilde{Y}\right]^{J}$ with respect to the coordinates $\left(x^{b}, x^{\beta}, x^{\bar{\beta}}\right)$ on $t\left(B_{m}\right)$, then we have

$$
\left[{ }^{c c} \widetilde{X},{ }^{c c} \widetilde{Y}\right]^{J}=\left({ }^{c c} \widetilde{X}\right){ }^{I} \partial_{I}\left({ }^{c c} \widetilde{Y}\right)^{J}-\left({ }^{c c} \widetilde{Y}\right)^{I} \partial_{I}\left({ }^{c c} \widetilde{X}\right)^{J} .
$$


Firstly, if $J=b$, we have

$$
\begin{aligned}
{\left[{ }^{c c} \widetilde{X},{ }^{c c} \widetilde{Y}\right]^{b} } & =\left({ }^{c c} \widetilde{X}\right)^{I} \partial_{I}\left({ }^{c c} \widetilde{Y}\right)^{b}-\left({ }^{c c} \widetilde{Y}\right)^{I} \partial_{I}\left({ }^{c c} \widetilde{X}\right)^{b} \\
& =\left({ }^{c c} \widetilde{X}\right)^{a} \underbrace{\partial_{a}\left({ }^{c c} \widetilde{Y}\right)^{b}}_{0}+\left({ }^{c c} \widetilde{X}\right)^{\alpha} \partial_{\alpha}\left({ }^{c c} \widetilde{Y}\right)^{b}+\left({ }^{c c} \widetilde{X}\right)^{\bar{\alpha}} \underbrace{\partial_{\alpha}\left({ }^{c c} \widetilde{Y}\right)^{b}}_{0}-\left({ }^{c c} \widetilde{Y}\right)^{a} \underbrace{\partial_{a}\left({ }^{c c} \widetilde{X}\right)^{b}}_{0}-\left({ }^{c c} \widetilde{Y}\right)^{\alpha} \partial_{\alpha}\left({ }^{c c} \widetilde{X}\right)^{b}-\left({ }^{c c} \widetilde{Y}\right)^{\bar{\alpha}} \underbrace{\partial_{\alpha}\left({ }^{c c} \widetilde{X}\right)^{b}}_{0} \\
& =\left({ }^{c c} X\right)^{\alpha} \partial_{\alpha}\left({ }^{c c} Y\right)^{b}-\left({ }^{c c} Y\right)^{\alpha} \partial_{\alpha}\left({ }^{c c} X\right)^{b} \\
& =X^{\alpha} \partial_{\alpha} \widetilde{Y}^{b}-Y^{\alpha} \partial_{\alpha} \widetilde{X}^{b} \\
& =\widetilde{X, Y]}
\end{aligned}
$$

by virtue of (4). Secondly, if $J=\beta$, we have

$$
\begin{aligned}
{\left[{ }^{c c} \widetilde{X},{ }^{c c} \widetilde{Y}\right]^{\beta} } & =\left({ }^{c c} \widetilde{X}\right)^{I} \partial_{I}\left({ }^{c} \widetilde{Y}\right)^{\beta}-\left({ }^{c c} \widetilde{Y}\right)^{I} \partial_{I}\left({ }^{c c} \widetilde{X}\right)^{\beta} \\
& =\left({ }^{c c} \widetilde{X}\right)^{a} \underbrace{\left.\partial_{a}{ }^{c c} \widetilde{Y}\right)^{\beta}}_{0}+\left({ }^{c c} \widetilde{X}\right)^{\alpha} \partial_{\alpha}\left({ }^{c c} \widetilde{Y}\right)^{\beta}+\left({ }^{c c} \widetilde{X}\right)^{\bar{\alpha}} \underbrace{\partial_{\bar{\alpha}\left({ }^{c c} \widetilde{Y}\right)^{\beta}}}_{0}-\left({ }^{c c} \widetilde{Y}\right)^{a} \underbrace{\partial_{a}\left({ }^{c} \widetilde{X}\right)^{\beta}}_{0}-\left({ }^{c c} \widetilde{Y}\right)^{\alpha} \partial_{\alpha}\left({ }^{c c} \widetilde{X}\right)^{\beta}-\left({ }^{c c} \widetilde{Y}\right)^{\bar{\alpha}} \underbrace{\partial_{\bar{\alpha}}\left({ }^{c c} \widetilde{X}\right)^{\beta}}_{0} \\
& =\left({ }^{c c} \widetilde{X}\right)^{\alpha} \partial_{\alpha}\left({ }^{c c} \widetilde{Y}\right)^{\beta}-\left({ }^{c c} \widetilde{Y}\right)^{\alpha} \partial_{\alpha}\left({ }^{c c} \widetilde{X}\right)^{\beta} \\
& =X^{\alpha} \partial_{\alpha} Y^{\beta}-Y^{\alpha} \partial_{\alpha} X^{\beta} \\
& =[X, Y]^{\beta}
\end{aligned}
$$

by virtue of (4). Thirdly, if $J=\bar{\beta}$, then we have

$$
\begin{aligned}
& {\left[{ }^{c c} \widetilde{X},{ }^{c c} \widetilde{Y}\right]^{\bar{\beta}}=\left({ }^{c c} \widetilde{X}\right)^{I} \partial_{I}\left({ }^{c c} \widetilde{Y}\right)^{\bar{\beta}}-\left({ }^{c c} \widetilde{Y}\right)^{I} \partial_{I}\left({ }^{c c} \widetilde{X}\right)^{\bar{\beta}}} \\
& =\left({ }^{c c} \widetilde{X}\right)^{a} \underbrace{\partial_{a}\left({ }^{c c} \widetilde{Y}\right)^{\bar{\beta}}}_{0}+\left({ }^{c c} \widetilde{X}\right)^{\alpha} \partial_{\alpha}\left({ }^{c c} \widetilde{Y}\right)^{\bar{\beta}}+\left({ }^{c c} \widetilde{X}\right)^{\bar{\alpha}} \partial_{\bar{\alpha}}\left({ }^{c} \widetilde{Y}\right)^{\bar{\beta}}-\left({ }^{c c} \widetilde{Y}\right)^{a} \underbrace{\partial_{a}\left({ }^{c c} \widetilde{X}\right)^{\bar{\beta}}}_{0}-\left({ }^{c c} \widetilde{Y}\right)^{\alpha} \partial_{\alpha}\left({ }^{c c} \widetilde{X}\right)^{\bar{\beta}}-\left({ }^{c c} \widetilde{Y}\right)^{\bar{\alpha}} \partial_{\bar{\alpha}}\left({ }^{c c} \widetilde{X}\right)^{\bar{\beta}} \\
& =X^{\alpha} \partial_{\alpha}\left(\sum_{\lambda=1}^{p} t_{\beta_{1} \ldots \beta_{q}}^{\alpha_{1} \ldots \varepsilon \ldots \alpha_{p}} \partial_{\varepsilon} Y^{\beta_{\lambda}}-\sum_{\mu=1}^{q} t_{\beta_{1} \ldots \varepsilon \ldots \beta_{q}}^{\alpha_{1} \ldots \alpha_{p}} \partial_{\beta_{\mu}} Y^{\varepsilon}\right) \\
& +\left(\sum_{\lambda=1}^{p} t_{\beta_{1} \ldots \beta_{q}}^{\alpha_{1} \ldots \ldots \alpha_{p}} \partial_{\varepsilon} X^{\alpha_{\lambda}}-\sum_{\mu=1}^{q} t_{\beta_{1} \ldots \gamma \ldots \beta_{q}}^{\alpha_{1} \ldots \alpha_{p}} \partial_{\alpha_{\mu}} X^{\gamma}\right) \partial_{\bar{\alpha}}\left(\sum_{\lambda=1}^{p} t_{\beta_{1} \ldots \beta_{q}}^{\alpha_{1} \ldots \sigma \ldots \alpha_{p}} \partial_{\sigma} Y^{\beta_{\lambda}}-\sum_{\mu=1}^{q} t_{\beta_{1} \ldots \gamma \ldots \beta_{q}}^{\alpha_{1} \ldots \alpha_{p}} \partial_{\beta_{\mu}} Y^{\gamma}\right) \\
& -Y^{\alpha} \partial_{\alpha}\left(\sum_{\lambda=1}^{p} t_{\beta_{1} \ldots \beta_{q}}^{\alpha_{1} \ldots \varepsilon \ldots \alpha_{p}} \partial_{\varepsilon} X^{\beta_{\lambda}}-\sum_{\mu=1}^{q} t_{\beta_{1} \ldots \varepsilon \ldots \beta_{q}}^{\alpha_{1} \ldots \alpha_{p}} \partial_{\beta_{\mu}} X^{\varepsilon}\right) \\
& -\left(\sum_{\lambda=1}^{p} t_{\beta_{1} \ldots \beta_{q}}^{\alpha_{1} \ldots \varepsilon \alpha_{p}} \partial_{\varepsilon} Y^{\alpha_{\lambda}}-\sum_{\mu=1}^{q} t_{\beta_{1} \ldots \varepsilon \ldots \beta_{q}}^{\alpha_{1} \ldots \alpha_{p}} \partial_{\beta_{\mu}} Y^{\varepsilon}\right) \partial_{\bar{\alpha}}\left(\sum_{\lambda=1}^{p} t_{\beta_{1} \ldots \beta_{q}}^{\alpha_{1} \ldots \sigma \ldots \alpha_{p}} \partial_{\sigma} X^{\beta_{\lambda}}-\sum_{\mu=1}^{q} t_{\beta_{1} \ldots \gamma \ldots \beta_{q}}^{\alpha_{1} \ldots \alpha_{p}} \partial_{\beta_{\mu}} X^{\gamma}\right) \\
& =X^{\alpha} \partial_{\alpha}\left(\sum_{\lambda=1}^{p} t_{\beta_{1} \ldots \beta_{q}}^{\alpha_{1} \ldots \alpha_{p}} \partial_{\varepsilon} Y^{\beta_{\lambda}}\right)-X^{\alpha} \partial_{\alpha} \sum_{\mu=1}^{q} t_{\beta_{1} \ldots \varepsilon \ldots \beta_{q}}^{\alpha_{1} \ldots \alpha_{p}}\left(\partial_{\beta_{\mu}} Y^{\varepsilon}\right) \\
& +\sum_{\lambda=1}^{p} t_{\beta_{1} \ldots \beta_{q}}^{\alpha_{1} \ldots \alpha_{p} \ldots \alpha_{p}} \partial_{\varepsilon} X^{\alpha_{\lambda}} \underbrace{\partial_{\bar{\alpha}} \sum_{\lambda=1}^{p} t_{\beta_{1} \ldots \beta_{q}}^{\alpha_{1} \ldots \sigma \ldots \alpha_{p}}}_{\delta_{\alpha_{\lambda}}^{\sigma}} \partial_{\sigma} Y^{\beta_{\lambda}}-Y^{\alpha} \partial_{\alpha}\left(\sum_{\lambda=1}^{p} t_{\beta_{1} \ldots \beta_{q}}^{\alpha_{1} \ldots \varepsilon \ldots \alpha_{p}} \partial_{\varepsilon} X^{\beta_{\lambda}}\right)+Y^{\alpha} \partial_{\alpha} \sum_{\mu=1}^{q} t_{\beta_{1} \ldots \varepsilon \ldots \beta_{q}}^{\alpha_{1} \ldots \alpha_{p}}\left(\partial_{\beta_{\mu}} X^{\varepsilon}\right)
\end{aligned}
$$

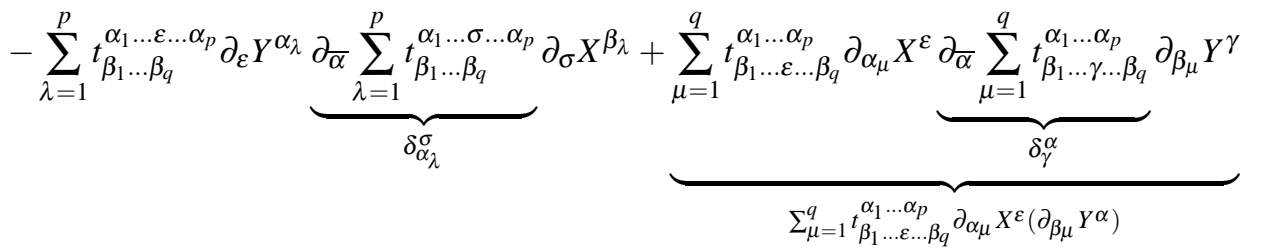




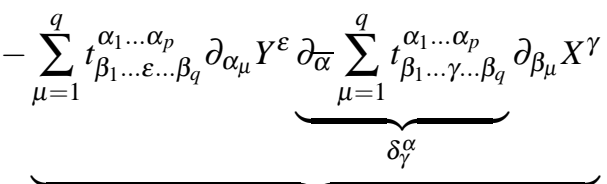

$$
\begin{aligned}
& \sum_{\mu=1}^{q} t_{\beta_{1} \ldots \varepsilon \ldots \beta q}^{\alpha_{1} \ldots \alpha_{p}} \partial_{\alpha_{\mu}} Y^{\varepsilon}\left(\partial_{\beta_{\mu}} X^{\alpha}\right) \\
& =\sum_{\lambda=1}^{p} t_{\beta_{1} \ldots \beta_{q}}^{\alpha_{1} \ldots \varepsilon \ldots \alpha_{p}}\left(\partial_{\varepsilon} X^{\sigma}\right)\left(\partial_{\sigma} Y^{\beta_{\lambda}}\right)+\sum_{\lambda=1}^{p} t_{\beta_{1} \ldots \beta_{q}}^{\alpha_{1} \ldots \varepsilon \ldots \alpha_{p}} X^{\alpha} \partial_{\alpha} \partial_{\varepsilon} Y^{\beta_{\lambda}}-\sum_{\lambda=1}^{p} t_{\beta_{1} \ldots \beta_{q}}^{\alpha_{1} \ldots \varepsilon \ldots \alpha_{p}}\left(\partial_{\varepsilon} Y^{\sigma}\right)\left(\partial_{\sigma} X^{\beta_{\lambda}}\right)
\end{aligned}
$$

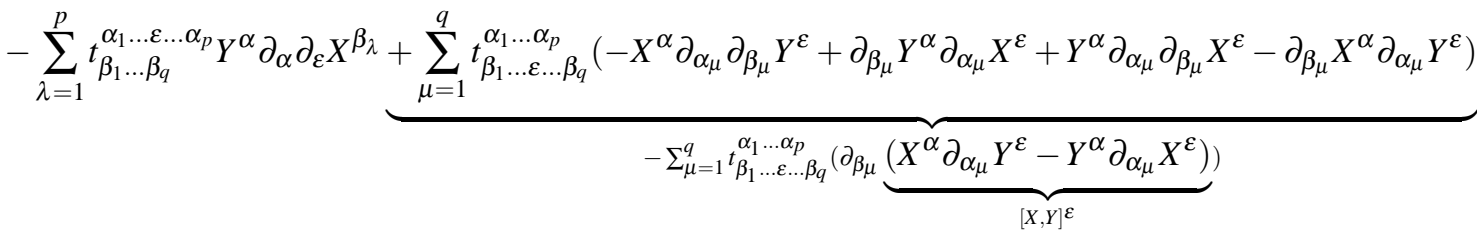

$$
\begin{aligned}
& =\sum_{\lambda=1}^{p} t_{\beta_{1} \ldots \beta_{q}}^{\alpha_{1} \ldots \varepsilon \ldots \alpha_{p}} \partial_{\varepsilon}[X, Y]^{\beta_{\lambda}}-\sum_{\mu=1}^{q} t_{\beta_{1} \ldots \varepsilon \ldots \beta_{q}}^{\alpha_{1} \ldots \alpha_{p}}\left(\partial_{\beta_{\mu}}[X, Y]^{\varepsilon}\right)
\end{aligned}
$$

by virtue of (4). On the other hand, we know that $c c \widetilde{[X, Y]}$ have components

$$
{ }^{c c} \widetilde{[X, Y]}=\left(\begin{array}{l}
\widetilde{[X, Y]}^{b} \\
{[X, Y]^{\beta}} \\
\sum_{\lambda=1}^{p} t_{\beta_{1} \ldots \beta_{q}}^{\alpha_{1} \ldots \varepsilon \ldots \alpha_{p}} \partial_{\varepsilon}[X, Y]^{\beta_{\lambda}}-\sum_{\mu=1}^{q} t_{\beta_{1} \ldots \varepsilon \ldots \beta_{q}}^{\alpha_{1} \ldots \alpha_{p}} \partial_{\beta_{\mu}}[X, Y]^{\varepsilon}
\end{array}\right)
$$

with respect to the coordinates $\left(x^{b}, x^{\beta}, x^{\bar{\beta}}\right)$ on $t_{q}^{p}\left(B_{m}\right)$. Thus Theorem 4 is proved.

\section{Acknowledgment}

The authors would like to thanks the referees for useful and improving comments.

\section{Competing interests}

The authors declare that they have no competing interests.

\section{Authors' contributions}

All authors have contributed to all parts of the article. All authors read and approved the final manuscript.

\section{References}

[1] T.V. Duc, Structure presque-transverse. J. Diff. Geom., 14 (1979), no. 2, 215-219.

[2] H. Fattaev, The Lifts of Vector Fields to the Semitensor Bundle of the Type (2, 0), Journal of Qafqaz University, 25 (2009), no. 1, 136-140.

[3] D. Husemoller, Fibre Bundles. Springer, New York, 1994.

[4] V. Ivancevic and T. Ivancevic, Applied Differential Geometry, A Modern Introduction, World Scientific, Singapore, 2007.

[5] H.B. Lawson and M.L. Michelsohn, Spin Geometry. Princeton University Press., Princeton, 1989.

[6] A. Salimov, Tensor Operators and their Applications. Nova Science Publ., New York, 2013. 
[7] A. A. Salimov and E. Kadıoğlu, Lifts of derivations to the semitangent bundle, Turk J. Math. 24 (2000), no. 3, $259-266$.

[8] N. Steenrod, The Topology of Fibre Bundles. Princeton University Press., Princeton, 1951.

[9] V. V. Vishnevskii, Integrable affinor structures and their plural interpretations. Geometry, 7.J. Math. Sci. (New York) 108 (2002), no. 2, 151-187.

[10] G. Walschap, Metric Structures in Differential Geometry, Graduate Texts in Mathematics, Springer-Verlag, New York, 2004.

[11] F. Yıldırım, On a special class of semi-cotangent bundle, Proc. Inst. Math. Mech. Natl. Acad. Sci. Azerb., 41 (2015), no. 1, $25-38$.

[12] F. Yıldırım, A pull-Back bundle of tensor bundles defined by projection of the tangent bundle, Ordu University Journal of Science and Technology, 7 (2017), no. 2, 353-366.

[13] F. Yildırım, Complete lift of a tensor field of type $(1,2)$ to semi-cotangent bundle, New Trends in Mathematical Sciences, 5 (2017), no. 4, 261-270.

[14] F. Yildirım, Note on the cross-section in the semi-tensor bundle, New Trends in Mathematical Sciences, 5 (2017), no. 2, $212-221$.

[15] F. Yildirim, M.B. Asl, F. Jabrailzade, Vector and affinor fields on cross-sections in the semi-cotangent bundle, Proc. Inst. Math. Mech. Natl. Acad. Sci. Azerb., 43 (2017), no. 2, 305-315.

[16] F. Yıldırım and A. Salimov, Semi-cotangent bundle and problems of lifts. Turk J Math, 38 (2014), no.2, 325-339. 